\title{
Does owning a pet protect older people against loneliness?
}

Jitka Pikhartova ${ }^{1 *}$, Ann Bowling $^{2}$ and Christina Victor ${ }^{1}$

\begin{abstract}
Background: Pet ownership is thought to make a positive contribution to health, health behaviours and the general well-being of older people. More specifically pet ownership is often proposed as a solution to the problem of loneliness in later life and specific 'pet based' interventions have been developed to combat loneliness. However the evidence to support this relationship is slim and it is assumed that pet ownership is a protection against loneliness rather than a response to loneliness. The aim of this paper is to examine the association between pet ownership and loneliness by exploring if pet ownership is a response to, or protection against, loneliness using Waves $0-5$ from the English Longitudinal Study of Ageing (ELSA).

Methods: Using data from 5,210 men and women in the English Longitudinal Study of Ageing, cross-sectional and longitudinal regression analysis was used to assess the bi-directional relationship between loneliness and pet ownership among adults aged $50+$.

Results: In 2001 (wave 0) 41\% of participants were pet owners compared with 30\% in 2010 (Wave 5). The association between pet ownership and loneliness is stronger in women than men, and in both directions (i.e. pet ownership predicting loneliness and loneliness predicting pet ownership) and of the similar magnitude (OR 1.2-1.4). Age, social relationships, demographic factors and health behaviour variables have only a minimal influence upon the association between loneliness and pet ownership. The results of our longitudinal analysis showed that women who reported being lonely always in Waves 0 to 5 were more likely to have a pet in Wave 5 .

Conclusion: Reported loneliness is dependent on socio-demographic characteristics such as gender, household income, household living arrangements and health status. Taking those factors into account, owning a pet significantly influences later reporting of loneliness in women in our longitudinal analysis. In the reverse direction, reported loneliness influences pet ownership in later waves. In both directions, the relatively strong gender interaction suggests the association is limited to women with effects for men minimal or non-existent.
\end{abstract}

Keywords: Loneliness, ELSA, Pet ownership, Longitudinal study, Old people

\section{Background}

Loneliness in later life, its prevalence and risk factors, has long been a focus of research. According to cognitive discrepancy theory, loneliness is defined as an unwanted discrepancy between desired and achieved levels of social contact [1].

In North America, Australasia and Western Europe research has consistently reported the prevalence of severe loneliness of approximately $10 \%$ for those aged 65 years and older with a further $30 \%$ classified as

\footnotetext{
* Correspondence: jitka.pikhartova@brunel.ac.uk

${ }^{1}$ Department of Clinical Sciences, College of Health and Life Sciences, Brunel University London, Uxbridge, Middlesex UB8 3PH, UK

Full list of author information is available at the end of the article
}

moderately lonely whilst countries in Central and Eastern Europe report prevalence rates of severe loneliness of between $15 \%$ and $20 \%$ [2]. Loneliness has been shown to be associated with a range of negative health outcomes and health behaviours (which vary between different age groups) $[3,4]$.

Previous research has identified a range of risk factors for the onset of loneliness which also vary somewhat across age groups. Predictors of loneliness in younger ages have been summarized by Mahon et al [5]. A much wider range of risk factors have been identified for older adults including gender [6], being widowed or divorced [7], reporting poorer self-rated health than expected $[8,9]$, 
sensory impairments [10], disability/impaired mobility [11], poverty and low material resources [6], time spent alone and household composition [8]. Living arrangements, social resources and social participation have been identified as potential mediators between health status and loneliness [12]. In terms of health outcomes loneliness has been linked with cardiovascular disease [13,14], depression [15] and Alzheimer disease [16], and has been proposed as a mortality accelerator [17-19]. According to review published by Holt-Lundstad et al. [20] the mortality excess associated with weak social relationships is similar to other established risk factors such as low physical activity, smoking or drinking. Loneliness is, therefore, an important public health issue, and it is thus important to identify factors which can protect against or reduce vulnerability to loneliness as a means of developing appropriate interventions. Some of the established risk factors have been used in interventions to reduce loneliness. Since 2000 at least five reviews and meta-analyses to evaluate the evidence for the effectiveness of interventions to reduce loneliness have been published. Four focussed on older adults (the reviews by Cattan, et al. [21], Findlay [22], Choi et al. [23] and Hagan et al. [24]) whilst Masi et al. [25] included adults of all ages. These reviews showed that only a limited number of interventions demonstrated any significant impact upon levels of loneliness [21].

A number of interventions attempting to prevent or reduce loneliness have used 'pet therapy' based upon the attachment theory of Bowlby which emphasized the human need to be attached to somebody, to be close, to form and maintain relationships and the need for a sense of belonging [26]. Such interventions also build on work by Lazarrus and Folkman [27] indicating that human-pet attachment could provide a unique and affordable source of social support [28]. In the UK it is estimated that there are approximately 27 million pets and $45 \%$ of British households own a pet (Pet Food Manufacturers Association; www.pfma.org.uk/pet-population/; accessed December 2013). The UK ranks second in Europe for dog ownership, and third for cat ownership [29]. It is claimed that people who own pets do so to improve their subjective well-being, for company and to feel loved, depended upon and wanted [30].

The evidence base to support the use of such pet-based interventions is weak. There are a number of studies focusing on pet ownership or on animal assisted therapy (AAT) for older adults, for adults with serious mental health problems [31], cardiovascular events [28,32,33], or living in care-homes [34] which demonstrate positive outcomes [35-37]. Several studies have explored how, and to what extent, feeling of loneliness and social exclusion can be remediated (or prevented) by pet ownership [38-40] based on the premise that this reduces the impact of stressors in everyday live and consequently symptoms of depression or anxiety [41-44]. It is also hypothesised that pets may substitute for missing attachment figure(s). However, those who are highly attached to their pet report higher levels of loneliness compared to those who do not have such close relationship with their pet [45].

The evidence for the beneficial impact of pet ownership on loneliness is inconsistent as some studies show no impact of pet-ownership on health status or on mortality [46]. Furthermore most studies looking at this relationship are cross sectional in design. There is lack of evidence from longitudinal and intervention studies of using animals to reduce loneliness and social isolation among older people [47] although some effective interventions have been identified [48]. Longitudinal studies examining the relationship between pet-ownership or frequent contact with animals, and health, well-being or loneliness are rare. Raina et al. [49], focusing on the relationship between pet ownership and the physical and mental health of older people, reported that those who owned a pet were more active at the end of the study period than non-pet-owners, and that pet ownership significantly modified the relationship between social support and change in mental health. [49] Guest et al. reported that hearing dogs had a big impact on reducing loneliness among hearing-impaired owners but they did not use any control group [50].

Given that the evidence is mixed and extremely limited, the effectiveness of the presence of home pets on the prevention of loneliness and social isolation and improvement of subjective well-being has been questioned [51-53]. Furthermore rather than pet ownership mediating against loneliness, it has been proposed that the true nature of the relationship is, in fact, reversed i.e. pet ownership is a response to loneliness. However there are few longitudinal studies assessing the relationship between loneliness and pet ownership in both directions (pet ownership as a response to loneliness or pet ownership as a protection against loneliness). [54] The aim of this paper is to contribute to the evidence examining the relationship between pet ownership and loneliness. We examine if pet ownership is a response to, or protection against, loneliness using the English Longitudinal Study of Ageing (ELSA) by considering four questions:

Question 1: Can pet ownership protect against future loneliness?

Question 2: Is current pet ownership a response to previous feelings of loneliness?

Question 3: How do different pathways of loneliness influence current pet ownership?

Question 4: What role do socio-demographic characteristics, known to be connected with loneliness, play in the association between pet ownership and loneliness? 
The answers to these questions will extend the evidence about the potential long-term effects of the presence of domestic animals in the lives of older people on the feelings of loneliness and vice versa.

\section{Methods \\ Data}

The analysis was performed on a subsample of publicly available data from the English Longitudinal Study of Ageing (ELSA). The ELSA dataset is based on the Health Survey for England (HSE) and is designed as a representative sample of the population aged $50+$ years of age living in the community in England. Those aged 50+ who participated in the HSE in 1998, 1999 and 2001 (referred to as Wave 0) were invited to participate in the Wave 1 of ELSA in 2002. The study has collected data every two years since 2002 with biological samples taken every 4 years. Participants gave full informed written consent to participate in the study and ethical approval was obtained from the London Multicentre Research Ethics Committee. More details about ELSA can be found at http://www.ifs.org.uk/elsa/documentation.php.

The two possible directions of the association between loneliness and pet ownership (as a protection against or response to loneliness and current pet ownership as a response to previous feelings of loneliness) are explored using two analytical samples. Questions about pet ownership were included in part of Wave 0 and in Wave 5 while questions related to loneliness were first included in Wave 2 and have been presented in all subsequent waves.

To answer Question 1 (does pet ownership protect against loneliness), we consider the relationship between pet-ownership in Wave 0 and loneliness reported in following waves (Waves 2 to 5). For this analysis a subset of 2,141 individuals present in the part of Wave 0 that included pet ownership questions (only 1 of the 3 years of HSE data that formed the original sample for ELSA) and subsequent ELSA waves is used.

To answer question 2 (is pet ownership a response to loneliness), we consider reported pet ownership in Wave 5 (the only other ELSA wave including pet ownership questions) and loneliness reported in Waves 2-5 using both cross-sectional and longitudinal analysis. The crosssectional analysis is based on data from Wave 5 (as it is the only wave with data both on loneliness and pet ownership). Longitudinal analysis will assess the relationship between loneliness reported in Waves 2 to 5 and pet ownership in the Wave 5 . Both these analyses (longitudinal and cross-sectional) will be conducted using a subsample of 5,210 core study members who took part in all waves with valid data related to loneliness and pet ownership. When we adjust our analysis for pet ownership in Wave 0 the sample size reduces to 2,141 .
The answer to the Question 3 is investigated by creating loneliness pathways between Waves 2 to 5 and using this as an independent variable and pet ownership as the dependent variable. The role of socio-demographic risk factors for loneliness (Question 4) will be answered by developing multivariable models as part of our analysis of questions $1-3$.

\section{Variables \\ Loneliness}

In the English Longitudinal Study of Ageing loneliness is measured by the short form of the Revised University of California, Los Angeles (UCLA) loneliness scale in Waves 2 to 5 . This instrument is a well-documented and widely used [55] and consists of three questions "How often do you feel you lack companionship", "How often do you feel left out" and "How often do you feel isolated from others?" Responses are recorded on a 3-point Likert scale ranging from hardly ever/never, some of the time and often, resulting in a theoretical range of 3-9, with a higher score indicating greater loneliness. Score were dichotomised with those scoring 3-5 (three bottom quartiles) classified as "not lonely" and those with scores 6-9 (upper quartile) as "lonely" [11]. We used the short form UCLA loneliness scale in preference to a single-item loneliness measure (one question from CES-D questionnaire "Have you felt lonely much of the time during the past week?" with answers yes/no) because of concerns about the reliability of this measure with older people [21] as they may mask feelings of loneliness as consequence of its stigmatization [56] but also because the question is focused on loneliness in the last week which can be misleading and a potential source of under- or over-reporting.

\section{Pet ownership}

Pet ownership in both Wave 0 and 5 was measured using responses to the question "Do you keep any household pets inside your house/flat?" followed by questions asking whether they had a dog, cat, bird, other furry pet and other pet.

\section{Covariates}

Gender, age, marital status $[57,58]$, the presence of close personal relationships (social networks), social participation, working status, social position, household income, and health status were used as covariates in the analysis. Marital status was dichotomised into those never married/ divorced/separated/widowed (not living with partner) and those living with partner (married/remarried/cohabiting). Information about social networks (family and friends) was available in all waves except Wave 0. A summary score was created to indicate whether the respondent had a close relationship with at least one of the following: spouse/partner, close family member or children and had 
at least one friend based upon responses to the following questions: about the number of family members and friends with whom respondent had contact, about the proximity of respondent's marital relationship; and whether the positive support from the spouse, children, other relatives and friends was or was not present. Social participation was constructed as summary score from information about membership of any club, society, and church group or being an active member of neighbourhood community. Working status was derived from responses to questions about whether participants were (self-)employed, retired or did not work. For social status the short version of NS-SEC 3 category classification was used managerial/ professional, intermediate and routine/manual. Household income was used categorised into quintiles [6].

Health related variables were also included in the analysis. A measure of immobility was constructed from difficulty in walking more than 200 yards (Wave 0) and difficulty walking more than $1 / 4$ of mile (other waves) [59]. Sensory impairment in Wave 0, derived variables about vision and hearing problems, were collected as binary measure with options "has condition" and "no condition". In Waves 2 and 5 the answers to questions about impairments were on 5-point Likert scale, and dichotomised to be comparable with Wave 0. Self-rated health was classified using 5-point Likert score scale, dichotomised as good and poor health and used from the same waves as information about pet-ownership (Waves 0 and 5).

\section{Statistical methods}

Frequency tables were constructed to describe the distribution of categorical variables in the individual waves of ELSA used in our analysis and mean age calculated for men and women for individual study waves.

The logistic regression analysis has three steps following our research questions. First, the role of pet ownership in Wave 0 (and in Wave 5 for cross-sectional analysis) as possible risk factor for loneliness in Waves 2 to 5 has been evaluated. A binary measure of loneliness was used as the dependent variable and pet ownership together with further covariates used as independent variables. Second, to consider pet ownership is a response to previous feelings of loneliness, we assessed if reported loneliness in Waves 2 to 5 affects pet ownership in Wave 5 . Pet ownership was used as dependent variable and loneliness categorised as a binary variable the independent measure.

For both questions cross sectional analysis using data from Wave 5 was followed by prospective analysis in which the dependent variable was from later wave than independent variables. Thus for question 1 we looked at pet ownership in Wave 0 and loneliness at Wave 2, Wave 3, Wave 4 and Wave 5 (four separate prospective analyses). For question 2 three prospective and one cross-sectional analyses were conducted (loneliness in Wave 2, Wave 3, Wave 4, and Wave 5 and pet ownership in Wave 5). The number of prospective analyses was determined by the availability of data related to pet ownership and our measure of loneliness.

In step 3 the role of loneliness in future pet ownership was further assessed using pathways of loneliness. Pet ownership in Wave 5 was the dependent variable and loneliness pathway the independent variable. Our loneliness pathway was constructed as a combination of dichotomised UCLA loneliness variables in Waves 2, 3, 4 and 5, and a five-fold typology created: "always lonely", "never lonely", "pathway into loneliness", "pathway out of loneliness" and "fluctuating pathway".

In all three steps of our analysis, crude unadjusted odds ratio (OR) and 95\% confidence interval (95\% CI) were estimated, and this was followed by adjusted multivariable analysis to answer Question 4. Variables were tested as possible effect modifiers. Results are presented separately for men and women due to significant or borderline non-significant effect modification by sex. Missing data for the self-completed UCLA loneliness scale part of questionnaire ranged from 9.3\% in Wave 2 and $11.5 \%$ in Wave 5 and was $1 \%$ for pet ownership questions. As the proportion of missing data was low we did not use imputation to increase analytical sample size [60].

Statistical analyses were carried out using STATA version MP 13.0

\section{Results}

\section{Characteristics of the sample}

Our analytical sample of 5,210 individuals was slightly younger in the first two waves than the main ELSA sample (61.4 years in Wave 0 compared with 63.4 for the main sample) but had higher mean age in later waves than the whole sample because the main dataset was boosted by new participants in consecutive waves. The gender distribution of the whole sample and analytical subsample is similar across all waves (43\% of males and $57 \%$ of females) as is marital status $(68.4 \%$ of our sample was married/living with partner while it was $66.9 \%$ in the whole ELSA sample). The proportion of widowed participants increased and proportion of married, remarried and those living with partner decreased by about $5 \%$ over 10 years of the study. The proportion of employed and not employed changed substantially and differs by nearly $30 \%$ between Wave 0 and Wave 5 which reflects the withdrawal of participants from the labour market.

A small percentage, $12 \%$, of the sample had no children; 98\% who had children reported a close relationship with them. Approximately one third of participants did not have spouse or partner with $97 \%$ of those who did reporting that they had a close relationship with them. Approximately $7.5 \%$ of participants did not have immediate family, and 
among those who had immediate family, approximately $13 \%$ did not have any close contact with them. Between 4 and $6 \%$ respondents did not have any friends and those who had them, $6 \%$ reported they do not have close relationship with them

A little over one-third, 39\%, of ELSA participants owned pet while it was $41 \%$ in our sample. The rates of loneliness (as measured by the UCLA loneliness scale) increased very slightly from $18 \%$ to $20.6 \%$ over 10 years. Gender differences were stable over all the waves and rates of reported loneliness were about 7\% higher in women compared with men (Table 1).

\section{Question 1: pet ownership and later loneliness Does pet ownership protect against loneliness?}

In our cross-sectional analysis, those who reported petownership in Wave 5 were 1.24 times more likely to report loneliness at the same time (see Table 2). In the prospective analysis those who reported pet-ownership in Wave 0 were 1.25 to 1.31 more likely to report loneliness in later waves. When the analysis was stratified by gender, as this is an effect modifier, having a pet increased reported loneliness 1.4-1.8 times in females (Table 2) after adjustment for all co-variates (age, marital status, working status, social class, health status, social inclusion, close personal relationships, and household income). This association was statistically significant for all analyses for women. The gender interaction was statistically significant, however, only in cross-sectional analysis when loneliness and pet-ownership were both measured Wave 5 and in one prospective analysis (Wave 0 to Wave 4). Although non-significant, the gender difference in the association between pet ownership and reported loneliness is very consistent (significant association in women and no association in men). We hypothesise that the nonsignificant interaction is a consequence of the smaller sample size in prospective analysis based upon reported pet ownership in Wave 0 , where the number of participants is small compared to the other waves. Our analysis suggests that having a pet increased the likelihood of reporting loneliness among females in all adjusted analyses.

\section{Question 2: loneliness and later pet ownership Is current pet ownership a response to previous feelings of loneliness?}

In Wave 5 the odds of owning a pet were $25 \%$ higher for those who were lonely as compared to those who were not. This result is mainly accounted for by women who were lonely for whom the odds of owning a pet were almost 50\% higher than their non-lonely counterparts (OR 1.3 to 1.8 ). Stratifying by pet ownership in Wave 0 , the magnitude of the effect of loneliness on pet ownership in Wave 5 is larger among women who had pet at Wave 0 than among women who did not (for example, OR 2.02 and 1.52 for the relationship between loneliness at Wave 4 and pet ownership at Wave 5; not shown in the tables) but none of these interactions were significant. Therefore the relationship between loneliness and pet ownership in Wave 5 was adjusted for the pet ownership in Wave 0 (Table 3, "Adjusted 2"). Although the sex-specific effects are different (and of similar magnitude as "Adjusted 1") the gender interactions are no longer statistically significant (except cross-sectional analysis) perhaps reflecting the reduced size of our analytical sample due to the limited availability of pet ownership data in Wave 0 .

\section{Question 3: loneliness pathways and later pet ownership How do different pathways of loneliness influence current pet ownership?}

Our final analysis evaluated how different pathways of loneliness affect pet ownership in Wave 5. The results are presented separately for men and women and are similar to previous analysis: no differences in the odds of pet ownership between different groups of men but significant between women who reported loneliness on all occasions or who moved out of loneliness and those who never reported loneliness. Those who always reported loneliness or moved out of loneliness were more likely to have pet in Wave 5 and these patterns were consistent when adjusted for pet ownership at Wave 0. Those who were persistently lonely were 2.4 times more likely to have a pet in Wave 5 than those who never reported loneliness whilst those who moved out of loneliness were 1.8 times more likely to have a pet than the non-lonely reference group (Table 4).

\section{Discussion}

Levels of reported loneliness in ELSA are approximately $19 \%$ and these are roughly stable over time. Women reported loneliness more frequently than men (a differential of around 7\%), and this difference is also approximately stable over time and supports previous studies using the revised UCLA scale [61] but no other studies using other scales to measure loneliness which reports higher rates of loneliness for men [62]. Loneliness in ELSA is reported more frequently in comparison with European data from the SHARE study conducted across a range of European countries (more information on http://www.share-project. org/). The reported rates of loneliness in SHARE in European countries (measured by the same instrument as in ELSA study and in comparable years) are somewhat lower oscillating between 5\% (in Denmark or Switzerland), $13 \%$ in France and 18\% in Hungary [2] compared with $20 \%$ for ELSA. These differences in the prevalence of loneliness across Europe support the hypothesis that loneliness is culturally defined and is associated with expectations. For example the prevalence of loneliness in Greece is 
Table 1 Descriptive characteristics of study sample

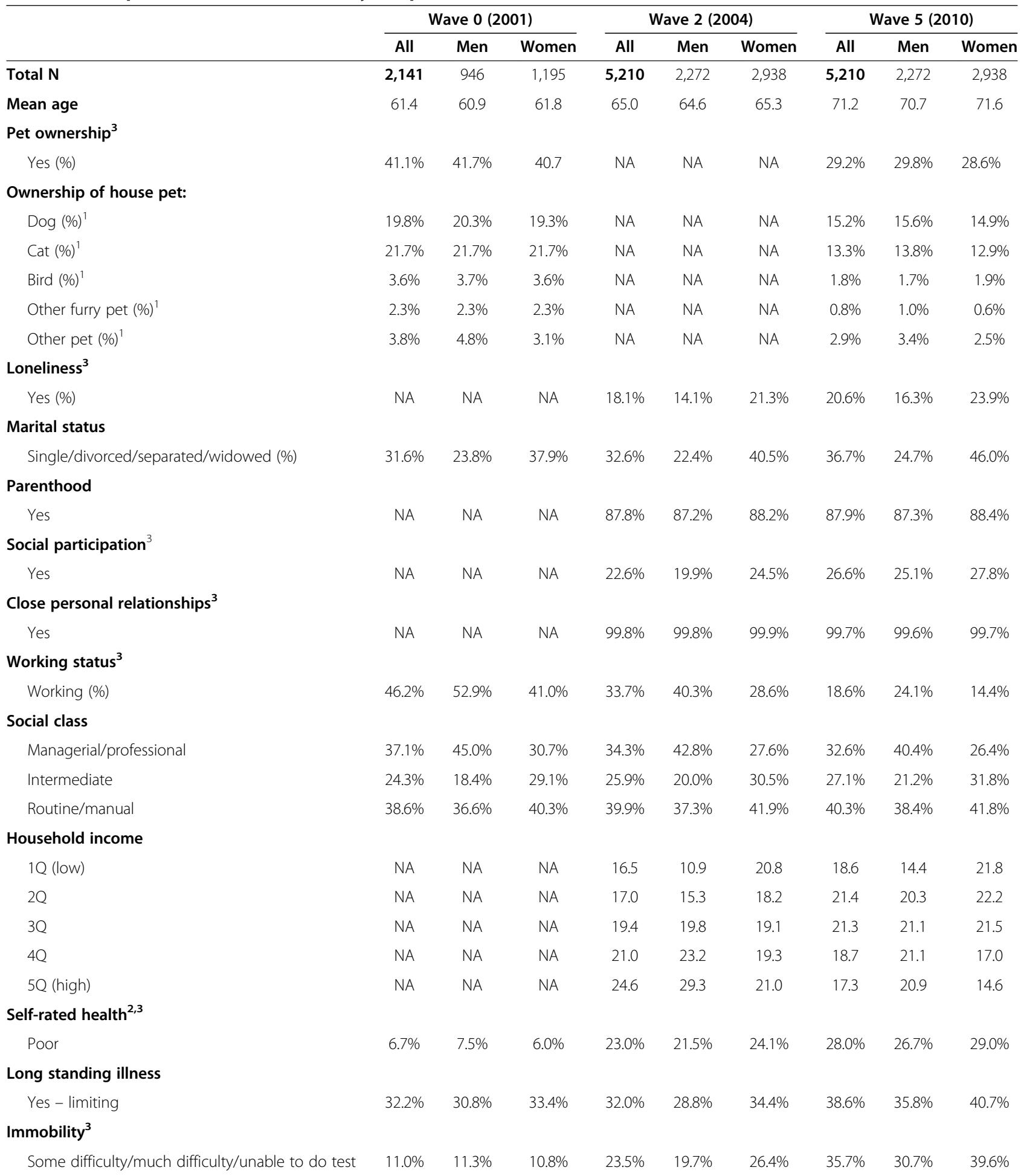


Table 1 Descriptive characteristics of study sample (Continued)

\begin{tabular}{|c|c|c|c|c|c|c|c|c|c|}
\hline \multicolumn{10}{|c|}{ Hearing difficulties $^{3}$} \\
\hline Yes & $6.7 \%$ & $8.5 \%$ & $5.3 \%$ & $4.2 \%$ & $5.7 \%$ & $3.1 \%$ & $5.6 \%$ & $7.1 \%$ & $4.5 \%$ \\
\hline \multicolumn{10}{|c|}{ Seeing difficulties ${ }^{3}$} \\
\hline Yes & $3.4 \%$ & $3.5 \%$ & 3.3 & $2.8 \%$ & $2.2 \%$ & $3.3 \%$ & $4.3 \%$ & $3.0 \%$ & $5.2 \%$ \\
\hline
\end{tabular}

traditionally reported as one of the highest in the Europe but only about $5 \%$ of older people live alone and about $60 \%$ reported that they had close daily contacts with family members or friends $[1,63]$.

The goal of our study was to assess the relationship between loneliness and having a pet. In particular we wanted to explore the direction of the association and answer the question whether pet ownership is a protection against or response to loneliness? Having a pet was reported by $41 \%$ of respondents at baseline (Wave 0 ) and by nearly $30 \%$ in Wave 5 and more than one pet by $24 \%$ and $18 \%$ of respondents respectively. We do not know why pet ownership decreased but it seems plausible that this may reflect reduced income resulting from retirement; deteriorating health resulting in pet care being too demanding and the death of pets.

We demonstrate that those who reported that they had a pet at the beginning of the study were 1.2-1.4 more likely to report loneliness compared to those who did not. This overall association masks a significant gender effect being confined to women but not men. Looking at the relationship the other way around and focusing upon loneliness as a predictor of pet ownership we see a similar association of a similar magnitude. In pooled analysis those who reported loneliness were 1.2-1.5 times more likely to have a pet at follow up. Again the gender interaction was significant in all analyses demonstrating that the association between loneliness and pet ownership is confined to women. Our pathway analysis demonstrated that women who always reported being lonely and those who moved out of loneliness were more likely to report pet ownership than their non-lonely contemporaries (odds ratios of 2.4 and 1.8 respectively). These results mean, perhaps, that pet ownership can be a response to loneliness for the always lonely and a protection for those who recovered from loneliness.

Gender is the key factor in our analysis. Our results suggest that the association between pet ownership and loneliness is particularly strong in women. Age, social, demographic and health behaviours variables including established risk factors for loneliness (such as age or marital status) do not substantially affect the magnitude or direction of the association between loneliness and pet ownership.

There are, of course, some limitations to our study. Loss to follow-up of individuals between the waves of ELSA data collection might have introduced selection bias. Recent articles using ELSA data suggest that

Table 2 The association between pet ownership (in Wave 0 and Wave 5) and odds of loneliness (in Waves 2 to 5) (OR and $95 \% \mathrm{Cl}$ )

\begin{tabular}{|c|c|c|c|c|c|}
\hline \multirow[b]{3}{*}{ Pet ownership } & \multicolumn{5}{|c|}{ Loneliness } \\
\hline & \multirow{2}{*}{$\begin{array}{c}\text { Cross-sectional } \\
\text { in wave } 5\end{array}$} & \multicolumn{4}{|c|}{ Prospective analysis } \\
\hline & & $\begin{array}{c}\text { Wave 0-wave } 2 \\
(2001-2004)\end{array}$ & $\begin{array}{c}\text { Wave 0-wave } 3 \\
(2001-2006)\end{array}$ & $\begin{array}{c}\text { Wave 0-wave } 4 \\
(2001-2008)\end{array}$ & $\begin{array}{c}\text { Wave } 0 \text {-wave } 5 \\
(2001-2010)\end{array}$ \\
\hline $\mathrm{N}$ & 4,638 & 1,958 & 1,886 & 1,853 & 1,890 \\
\hline \multicolumn{6}{|l|}{ All } \\
\hline No & 1 (ref) & 1 (ref) & 1 (ref) & 1 (ref) & 1 (ref) \\
\hline Yes & $1.24(1.06-1.47)$ & $1.25(0.98-1.61)$ & $1.23(0.96-1.58)$ & $1.38(1.08-1.77)$ & $1.31(1.03-1.68)$ \\
\hline \multicolumn{6}{|l|}{ Men } \\
\hline No & 1 (ref) & 1 (ref) & 1 (ref) & 1 (ref) & 1 (ref) \\
\hline Yes & $1.04(0.78-1.38)$ & $1.03(0.69-1.54)$ & $0.98(0.65-1.49)$ & $0.84(0.55-1.29)$ & $1.06(0.70-1.60)$ \\
\hline \multicolumn{6}{|l|}{ Women } \\
\hline No & 1 (ref) & 1 (ref) & 1 (ref) & 1 (ref) & 1 (ref) \\
\hline Yes & $1.41(1.15-1.73)$ & $1.39(1.01-1.92)$ & $1.40(1.03-1.90)$ & $1.84(1.34-2.52)$ & $1.50(1.09-2.05)$ \\
\hline P sex interaction & 0.03 & 0.30 & 0.19 & 0.005 & 0.13 \\
\hline
\end{tabular}

Adjusted for gender (in pooled analysis), age, marital status, working status, social class, health status, social participation, close personal relationships, household income. 
Table 3 The association between reported loneliness (Waves 2 to 5) and odds of pet ownership (Wave 5) (OR and $95 \% \mathrm{Cl}$ )

\begin{tabular}{ccccc}
\hline & \multicolumn{4}{c}{ Pet ownership } \\
\cline { 2 - 5 } Loneliness & $\begin{array}{c}\text { Cross-sectional } \\
\text { Wave 5 }\end{array}$ & $\begin{array}{c}\text { Pave 2 to wave5 } \\
(\mathbf{2 0 0 4 - 2 0 1 0 )}\end{array}$ & $\begin{array}{c}\text { Wave 3 to wave 5 } \\
\text { (2006-2010) }\end{array}$ & $\begin{array}{c}\text { Wave 4 to wave 5 } \\
\text { (2008-2010) }\end{array}$ \\
\hline N & 4,638 & 4,743 & 4,640 & 4,571
\end{tabular}

Adjusted 1

All

$\begin{array}{ccccr}\text { No } & 1 \text { (ref) } & 1 \text { (ref) } & 1 \text { (ref) } & 1 \text { (ref) } \\ \text { Yes } & 1.24(1.05-1.46) & 1.20(1.01-1.42) & 1.21(1.02-1.43) & 1.45(1.23-1.72) \\ \text { Men } & 1 \text { (ref) } & 1 \text { (ref) } & 1 \text { (ref) } & 1 \text { (ref) } \\ \text { No } & 1.03(0.77-1.36) & 1.03(0.76-1.38) & 0.89(0.67-1.20) & 1.02(0.77-1.36) \\ \text { Yes } & 1(\mathrm{ref}) & 1 \text { (ref) } & 1 \text { (ref) } & 1 \text { (ref) } \\ \text { Women } & 1.40(1.14-1.72) & 1.30(1.05-1.61) & 1.42(1.16-1.74) & 1.76(1.43-2.17) \\ \text { No } & 0.006 & 0.02 & 0.001 & <0.001\end{array}$

Adjusted 2

All

No 1 (ref)

Yes $\quad 1.39(1.01-1.91)$

Men

No 1 (ref)

$\begin{array}{ll}\text { Yes } & 0.79(0.47-1.34)\end{array}$

Women

$\begin{array}{lcccc}\text { No } & 1 \text { (ref) } & 1 \text { (ref) } & 1 \text { (ref) } & 1 \text { (ref) } \\ \text { Yes } & 1.84(1.26-2.68) & 1.28(0.86-1.89) & 1.56(1.07-2.27) & 1.81(1.22-2.67) \\ \text { sex interaction } & 0.006 & 0.26 & 0.07 & 0.07\end{array}$

Adjusted 1 = for gender(in pooled analysis), age, marital status, working status, social class, health status, social participation, close personal relationships, household income.

Adjusted 2 = additionally adjusted for pet ownership in Wave $0, \mathrm{~N}=2,141$.

Table 4 Pathways of loneliness and pet ownership in Wave 5 (OR and 95\% Cl)

\begin{tabular}{|c|c|c|c|c|c|c|}
\hline \multirow{2}{*}{$\begin{array}{l}\text { Loneliness } \\
\text { pathway }\end{array}$} & \multirow{2}{*}{$\begin{array}{c}\text { Sample } \\
1(N)\end{array}$} & \multirow{2}{*}{$\begin{array}{l}\text { Sample } \\
2(N)^{1}\end{array}$} & \multicolumn{2}{|c|}{ Men } & \multicolumn{2}{|c|}{ Women } \\
\hline & & & $\begin{array}{l}\text { Adjusted- } \\
\text { sample } 1\end{array}$ & $\begin{array}{l}\text { Adjusted- } \\
\text { sample } 2\end{array}$ & $\begin{array}{l}\text { Adjusted- } \\
\text { sample } 1\end{array}$ & $\begin{array}{l}\text { Adjusted- } \\
\text { sample } 2\end{array}$ \\
\hline Never lonely & 2,540 & 1,025 & 1 (ref) & 1 (ref) & 1 (ref) & 1 (ref) \\
\hline Always lonely & 256 & 84 & $0.81(0.44-1.47)$ & $1.12(0.38-3.36)$ & $1.41(0.99-2.00)$ & $2.40(1.18-4.89)$ \\
\hline Into loneliness & 332 & 139 & $1.11(0.76-1.62)$ & $1.09(0.59-2.04)$ & $0.97(0.70-1.35)$ & $0.76(0.41-1.40)$ \\
\hline Out of loneliness & 376 & 165 & $0.94(0.60-1.48)$ & $0.70(0.31-1.60)$ & $1.71(1.25-2.35)$ & $1.81(1.02-3.20)$ \\
\hline Fluctuating & 333 & 137 & $1.02(0.67-1.55)$ & $1.00(0.45-2.21)$ & $1.24(0.88-1.73)$ & $1.28(0.70-2.34)$ \\
\hline
\end{tabular}

Adjusted 1 = for gender (in pooled analysis), for gender (in pooled analysis), age, marital status, working status, social class, health status, social participation, close personal relationships, household income.

Adjusted 2 =additionally adjusted for pet ownership in Wave $0=$ Sample 2.

${ }^{1}$ Those, who have information on pet ownership in Wave 0. 
sample attrition is, for example, greater among those who were in a disadvantaged socioeconomic position at the start of the study but any bias due to attrition might be only small [64]. There was a relatively small subsample of participants, who were asked about pet ownership in Wave 0 of ELSA. We do not have information about how long participants had owned a pet or whether they looked after somebody else's pet. We also do not have sufficient consecutive information to determine the relation between the initial reporting of loneliness, possible acquisition of a pet and the subsequent loneliness status to see the whole sequence of events to explore reverse causality in full detail. We could not adjust for seasonality and although loneliness is higher in spring and winter $[65,66]$ we do not think that this would significantly alter our results. Finally, as we performed relatively large number of hypothesis tests, we focused more on the magnitude of the effects when interpreting the results rather than just purely focusing on significance of findings.

\section{Conclusions}

It is commonly assumed that pet ownership 'protects' older people against loneliness. Our analysis has demonstrated that, for women, this may be a plausible hypothesis as it is associated with recovery from loneliness. However we have also demonstrated that for women who are always lonely pet ownership may be a response to their loneliness. Our results contribute to research on loneliness by demonstrating the complexity of the link between pet ownership and loneliness-it can be both a response to loneliness and a potential pathway out of loneliness. We also demonstrate that these relationships are only demonstrated by women and are not moderated by established loneliness risk factors or confounders.

These results suggest a number of areas for future research and have implications for policy and practice. There is considerable scope for qualitative research examining the issue of pet ownership in later life in more detail and how older people see this as a response or pathway out of loneliness. There is a clear need for such research to explore the important gender dimension identified in our analysis. Quantitative studies can demonstrate a link between gender, loneliness and pet ownership but we need to conduct qualitative research to explore the factors that account for these relationships. In addition these results caution us as to the appropriateness of pet based therapies and interventions against loneliness. We may speculate that, based on our findings, that such interventions may be more appropriate and acceptable to women than men. Again we need further research to explore the nature of the relationships between gender, loneliness and pet ownership in order to develop interventions that are appropriate, acceptable and effective.

\section{Abbreviations}

ELSA: English Longitudinal Study of Ageing; AAT: Animal assisted therapy; UCLA: University of California, Los Angeles; SHARE: The Survey of Health, Ageing and Retirement in Europe; OR: Odds ratio; Cl: Confidence interval.

\section{Competing interests}

The authors declare that they have no competing interests.

\section{Authors' contributions}

JP was responsible for developing the research idea, the data preparation and performing the statistical analysis. $\mathrm{AB}$ and $\mathrm{CV}$ have participated in the design and broadening the idea, and all authors participated in the preparation of the manuscript. All authors read and approved the final manuscript.

\section{Acknowledgment}

The research was funded by ESRC grant reference ES/K004077/1 as part of the Secondary Data Analysis Initiative. The authors thank ELSA participants and researchers; and ESDS for enabling use of ELSA data for this analysis.

\section{Author details}

${ }^{1}$ Department of Clinical Sciences, College of Health and Life Sciences, Brunel University London, Uxbridge, Middlesex UB8 3PH, UK. ${ }^{2}$ University of Southampton, Faculty of Health Sciences, Southampton SO17 1BJ, UK.

Received: 13 May 2014 Accepted: 16 September 2014

Published: 20 September 2014

\section{References}

1. Peplau LA, Perlman D: Perspectives on loneliness. In Loneliness; A sourcebook of current theory, research and therapy. Edited by Peplau LA, Perlman D. New York: A Willey-Interscience Publication; 1982:1-18.

2. Fokkema T, De Jong Gierveld J, Dykstra PA: Cross-national differences in older adult loneliness. J Psychol 2012, 146:201-228.

3. Stanley $I H$, Conwell Y, Bowen C, Van Orden KA: Pet ownership may attenuate loneliness among older adult primary care patients who live alone. Aging Ment Health 2014, 18:394-399.

4. Cacioppo JT, Hawkley LC, Crawford E, Ernst JM, Burleson MH, Kowalewski RB, Malarkey WB, Van Cauter E, Berntson GG: Loneliness and health: potential mechanisms. Psychosom Med 2002, 64:407-417.

5. Mahon NE, Yarcheski A, Yarcheski TJ, Cannella BL, Hanks MM: A meta-analytic study of predictors for loneliness during adolescence. Nursing Res 2006, 55:308-315.

6. Pinquart M, Sorensen S: Influences on loneliness in older adults: A meta-analysis. Basic App/ Soc Psychol 2001, 23:245-266.

7. Savikko N, Routasalo P, Tilvis RS, Strandberg TE, Pitkälä KH: Predictors and subjective causes of loneliness in an aged population. Arch Gerontol Geriatr 2005, 41:223-233.

8. Wenger GC, Davies R, Shahtahmasebi S, Scott A: Social isolation and loneliness in old age: Review and model refinement. Ageing and Society 1996, 16:333-358.

9. Victor C, Grenade L, Boldy D: Measuring loneliness in later life: a comparison of differing measures. Rev Clin Geront 2005, 15:63-70.

10. Wallhagen MI, Strawbridge WJ, Shema SJ, Kurata J, Kaplan GA: Comparative impact of hearing and vision impairment on subsequent functioning. J Am Geriatr Soc 2001, 49:1086-1092.

11. Steptoe A, Shankar A, Demakakos P, Wardle J: Social isolation, loneliness, all-cause mortality in older men and women. PNAS 2013, 110:5797-5801

12. Burholt V, Scharf T: Poor health and loneliness in later life: The role of depressive symptoms, social resources, and rural environments. J Gerontol Series B: Psychol Sci Soc Sci 2013, 69:311-324.

13. Thurston RC, Kubzansky LD: Women, loneliness, and incident coronary heart disease. Psychosom Med 2009, 71:836-842.

14. Udell JA, Steg PG, Scirica BM, Smith SC, Ohman ME, Eagle KA, Goto S, Cho Jl, Bhatt DL: Living alone and cardiovascular risk in outpatients at risk of or with atherothrombosis. Arch Intern Med 2012, 172:1086-1095.

15. Cacioppo JT, Hughes ME, Waite LJ, Hawkley LC, Thisted RA: Loneliness as a specific risk factor for depressive symptoms: cross-sectional and longitudinal analyses. Psychol Aging 2006, 21:140-151.

16. Wilson RS, Krueger KR, Arnold SE, Schneider JA, Kelly JF, Barnes LL, Tang Y, Bennett DA: Loneliness and risk of Alzheimer disease. Arch Gen Psychiatry 2007, 64:234-240. 
17. Olsen RB, Olsen J, Gunner-Svensson F, Waldstrom B: Social networks and longevity. A 14 year follow-up study ammong elderly in Denmark. Soc Sci Med 1991, 33:1189-1195.

18. Penninx BWJH, Van Tilburg T, Kriegsman DMW, Deeg DJH, Boeke AJP, Van Eijk M: Effects of social support and personal coping resources on mortality in older age: The Longitudinal Aging Study Amsterdam. Am J Epid 1997, 146:510-519.

19. Perissinotto CM, Cenzer IS, Covinsky KE: Loneliness in older persons: A predictor of functional decline and death. Arch Intern Med 2012, 172:1078-1083.

20. Holt-Lunstad J, Smith TB, Layton JB: Social relationships and mortality risk: a meta-analysis review. PLOS Medicine 2010, 7:e10000316.

21. Cattan $M$, White $M$, Bond J, Learmouth $A$ : Preventing social isolation and loneliness among older people: a systematic review of health promotion interventions. Ageing Soc 2005, 25:41-67.

22. Findlay RA: Interventions to reduce social isolation amongst older people: where is the evidence? Ageing Soc 2003, 23:647-658.

23. Choi M, Kong S, Jung D: Computer and internet interventions for loneliness and depression in older adults:A meta-analysis. Healthc Inform Res 2012, 18:191-198.

24. Hagan R, Manktelow R, Taylor BJ, Mallett J: Reducing loneliness amongst older people: a systematic search and narrative review. Aging Ment Health 2014, 18:683-693.

25. Masi CM, Chen HY, Hawkley LC, Cacioppo JT: A meta-analysis of interventions to reduce loneliness. Pers Soc Psychol Rev 2010, 15:219-266.

26. Bowlby J: The making and breaking of affectional bonds. I. Aetiology and psychopathology in the light of Attachment Theory. Brit J Psychiat 1977, 130:201-210.

27. Lazarus RS, Folkman S: Transactional theory and research on emotions and coping. Eur J Personality 1987, 1:141-169.

28. McNicholas J, Gilbey A, Rennie A, Ahmedzai S, Dono JA, Ormerod E: Pet ownership and human health: a brief review of evidence and issues. BMJ 2005, 331:1252-1254.

29. The European Pet Food Industry: Facts \& Figures 2012. Brussels: The European Pet Food Industry Federation; 2012.

30. Podberscek AL, Paul ES, Serpell JA: Companion Animals and us: Exploring relationships between people and pets. Cambridge: Cambridge University Press; 2005.

31. Siegel JM: Stressful life events and use of physician services among the elderly: the moderating role of pet ownership. J Personal Soc Psychol 1990, 58:1081-1086.

32. Friedmann E, Katcher AH, Lynch JJ, Thomas SA: Animal companion and one-year survival of patients after discharge from a coronary care unit. Public Health Reports 1980, 95:307-312.

33. Herrald MM, Tomaka J, Medina AY: Pet ownership predicts adherence to cardiovascular rehabilitation. J App/ Soc Psychol 2006, 32:1107-1123.

34. Banks MR, Banks WA: Animal-assisted therapy and loneliness in nursing homes: use of robotic versus living dogs. J Am Med Dir Assoc 2008, 9:173-177.

35. Jennings LB: Potential benefits of pet ownership in health promotion. J Holist Nurs 1997, 15:358-372.

36. Andersson L: Loneliness research and intervention: a review of the literature. Aging Ment Health 1998, 2:264-274.

37. Garrity TF, Stallones L: Effect of pet contact on human well-being: review of recent research. In Companion animals in human health. Edited by Wilson CC, Turner DC. London: Sage Publications, Inc; 1998.

38. Baumeister RF, Leary MR: The need to belong: desire for interpersonal attachments as a fundamental human motivation. Psychol Bull 1995 117:497-529.

39. McNicholas J, Collis GM: Dogs as catalysts for social interactions: robustness of the effect. Br J Psycholo 2000, 91:61-70.

40. Banks MR, Banks WA: The effects of animal-assisted therapy on loneliness in an elderly population in long-term care facilities. J Gerontology 2002, 57A:M428-M432.

41. Holbrook MB, Stephens LD, Day E, Holbrook SM, Strazar G: A collective stereographic photo essay on key aspects of animal companionship: the truth about dogs and cats. Acad Market Sci Rev 2001, 1:1-16.

42. Wells DL: The effects of animals on human health and well-being. J Soc Issues 2009, 65:523-543.

43. Kanamori M, Suzuki M, Tanaka M: Maintenance and improvement of quality of life among elderly patients using pet-type robot. Japanese $J$ Geriastrics 2002, 39:214-218.
44. Tamura T, Yonemitsu S, Oikawa D, Kawakami A, Higashi Y, Fujimooto T, Nakajima K: Is an entertainment robot useful in the care of elderly people with severe dementia? J Gerontol A Biol Sci Med Sci 2004, 59:M83-M85.

45. Antonacopoulos NM, Pychyl TA: An examination of the potential role of pet ownership, human social support and pet attachment in the psychological health of individuals living alone. Anthrozoos 2008, 21:139-152.

46. Gillum RF, Obisenan TO: Living with companion animals, physical activity and mortality in U.S. National Cohort. Int J Environ Res Public Health 2010, 7:2452-2459.

47. Cattan M, White M, Learmouth A, Bond J: Are services and activities for socially isolated and lonely older people accessible, equitable, and inclusive? Research. Policy and Planning 2005, 23:149-164.

48. Dickens AP, Richards SH, Greaves CJ, Campbell J: Interventions targeting social isolation in older people: a systematic review. BMC Public Health 2011, 11:647.

49. Raina P, Waltner-Toews D, Bonnett B, Woodward C, Abernathy T: Influence of companion animals on the physical and psychological health of older people: An analysis of one-year longitudinal study. J Am Geriatr Soc 1999, 47:323.

50. Guest CM, Collins GM, McNicholas J: Hearing dogs: A longitudinal study of social and psychological effects on deaf and hard-of-hearing recipients. $J$ Deaf Studies and Deaf Education 2006, 11:252-261.

51. Gilbey A, McNicholas J, Collis GM: A longitudinal test of the belief that companion animal ownership can help reduce loneliness. Anthrozoos 2007, 20:345-353.

52. Parslow RA, Jorm AF, Christensen $H$, Rodgers $B$, Jacomb P: Pet ownership and health in older adults: findings from survey of 2,551 community-based Australians aged 60-64. Gerontology 2005, 51:40-47.

53. Miltiades $\mathrm{H}$, Shearer J: Attachments to pet dog and depression in rural older adults. Anthrozoos 2011, 24:147-154.

54. Headey B, Grabka M: Pets and human health in Germany and Australia: national longitudinal results. Social Indicators Research 2007, 80:297-311.

55. Russel D, Peplau LA, Cutrona CE: The revised UCLA Loneliness Scale: Concurrent and discriminant validity evidence. J Personality Social Psychology 1980, 39:472-480.

56. Victor C, Scambler S, Bond J, Bowling A: Being alone in later life: loneliness, socila isolation and living alone. Rev Clin Geront 2000, 10:407-417.

57. Victor $C$, Bowling A: A longitudinal analysis of loneliness among older people in Great Britain. J Psych 2012, 146:313-331.

58. Victor C, Yang K: The prevalence of loneliness among adults: a case study of the United Kingdom. J Psych 2012, 146:85-104

59. Melzer D, Gardener E, Guralnik JM: Mobility disability in the middle-aged: cross-sectional associations in the English Longitudinal Study of Ageing. Age and Ageing 2005, 34:594-602.

60. Hippisley-Coc J, Coupland C, Vinogradova Y, Robson J, May M, Brindle P: Derivation and validation of QRISK, a new cardiovascular disease risk score for the United Kingdom: prospective open cohort study. BMJ 2007, 335:136.

61. Flaherty J, Richman J: Gender differences in the perception and utilization of social support: theoretical perspectives and an empirical test. Soc Sci Med 1989, 28:1221-1228.

62. Borys S, Perlman D: Genders differences in loneliness. Pers Soc Psychol Bull 1985, 11:63-74

63. Victor C, Scambler SJ, Shah S, Cook DG, Harris T, Rink E, De Wilde S: Has loneliness amongst older people increased? An investigation into variations between cohorts. Ageing Soc 2002, 22:585-597.

64. Demakakos $P$, Marmot M, Steptoe A: Socioeconomic position and the incidence of type 2 diabetes: the ELSA study. Eur J Epidemio/ 2012 27:367-378

65. Wenz FV: Seasonal suicide attempts and forms of loneliness. Psychological Reports 1977, 40:807-810.

66. Zhong CB, Leonardelli GJ: Cold and lonely: does social exclusion literally feel cold? Psychol Sci 2008, 19:839-842.

doi:10.1186/1471-2318-14-106

Cite this article as: Pikhartova et al:: Does owning a pet protect older people against loneliness? BMC Geriatrics 2014 14:106. 